\title{
Preoperative clinical characteristics scoring system for differentiating uterine leiomyosarcoma from fibroid
}

\author{
Guorui Zhang, Xin Yu*i(D, Lan Zhu, Qingbo Fan, Honghui Shi and Jinghe Lang
}

\begin{abstract}
Background: Morcellation may lead to intraperitoneal spread of tumor cells, thus making prognosis of undiagnosed uterine leiomyosarcoma (ULMS) worse. However, preoperative diagnosis of ULMS remains challenging. This study aimed to design a preoperative clinical characteristics scoring system for differentiating ULMS from uterine fibroid.

Methods: This study enrolled 45 ULMS patients and 180 uterine fibroid patients in Peking Union Medical College Hospital from January 2013 to December 2018.

Results: The incidence of occult ULMS was $0.59 \%$ (95\% Cl, 0.39-0.71\%). Age $\geq 40$ years old (OR $2.826,95 \% \mathrm{Cl} 1.326-$ 5.461), tumor size $\geq 7 \mathrm{~cm}$ (OR 6.930, 95\% Cl 2.872-16.724), neutrophil-to-lymphocyte ratio (NLR) $\geq 2.8$ (OR 3.032, $95 \% \mathrm{Cl} 1.288-7.13)$, number of platelet $\geq 298 \times 10^{9} / \mathrm{L}(\mathrm{OR} 3.688,95 \% \mathrm{Cl} 1.452-9.266)$ and lactate dehydrogenase $(\mathrm{LDH}) \geq 193 \mathrm{U} / \mathrm{L}$ (OR 6.479, 95\%Cl 2.658-15.792) were independent predictors of ULMS. A preoperative clinical characteristics scoring system was designed based on OR values, with a total score of 7 points. Tumor size $\geq 7 \mathrm{~cm}$, $L D H \geq 193 \mathrm{U} / \mathrm{L}$ were assigned 2 points, while age $\geq 40$ years old, NLR $\geq 2.8$ and number of platelet $\geq 298 \times 10^{9} / \mathrm{L}$ were assigned 1 point. Score $\geq 4$ points was a useful predictor in diagnosing ULMS from fibroid (sensitivity 0.800, specificity 0.778).

Conclusions: The incidence of occult ULMS was low. Age $\geq 40$ years old, tumor size $\geq 7 \mathrm{~cm}, L D H \geq 193 \mathrm{U} / \mathrm{L}, \mathrm{NLR} \geq$ 2.8 and number of platelet $\geq 298 \times 10^{9} / \mathrm{L}$ were independent predictors of ULMS. The preoperative clinical characteristics scoring system could be helpful in preoperative diagnosis of occult ULMS.
\end{abstract}

Keywords: Uterine leiomyosarcoma, Fibroid, Diagnosis

\section{Background}

Uterine fibroid is the most common benign tumor in gynecology, affecting $40-60 \%$ of all reproductive women, and is the main cause of hysterectomy worldwide. Minimally invasive surgeries, including myomectomy and hysterectomy, have advantages of low complication rate, mild pain and quick recovery. Morcellation provides

\footnotetext{
*Correspondence: yuxpumch@126.com

Department of Obstetrics and Gynecology, Peking Union Medical College Hospital, Peking Union Medical College, Chinese Academy of Medical Sciences, No. 1 Shuaifuyuan, Wangfujing, Dongcheng District, Beijing 100730, People's Republic of China
}

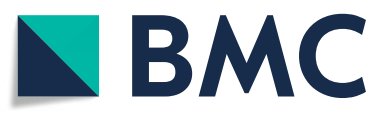

C C The Author(s). 2020 Open Access This article is licensed under a Creative Commons Attribution 4.0 International License, which permits use, sharing, adaptation, distribution and reproduction in any medium or format, as long as you give appropriate credit to the original author(s) and the source, provide a link to the Creative Commons licence, and indicate if changes were made. The images or other third party material in this article are included in the article's Creative Commons licence, unless indicated otherwise in a credit line to the material. If material is not included in the article's Creative Commons licence and your intended use is not permitted by statutory regulation or exceeds the permitted use, you will need to obtain permission directly from the copyright holder. To view a copy of this licence, visit http://creativecommons.org/licenses/by/4.0/. The Creative Commons Public Domain Dedication waiver (http://creativecommons.org/publicdomain/zero/1.0/) applies to the data made available in this article, unless otherwise stated in a credit line to the data. possibility of minimally invasive surgery in giant uterine fibroid. Uterine leiomyosarcoma (ULMS) is a rare uterine malignant tumor originating from smooth muscle cell of uterine myometrium, with high malignancy and poor prognosis. The 5-year overall survival rate for International Federation of Gynecology and Obstetrics (FIGO) stage III-IV ULMS is approximately 25-33\% [1]. Morcellation may lead to intraperitoneal spread of tumor cells, thus making prognosis of undiagnosed ULMS patients worse. Compared to open surgery group, incidence of peritoneal dissemination in stage I and II 
ULMS increases (44\% vs. $12.9 \%$ ) in morcellation group [2]. Besides, intraperitoneal recurrence rate of ULMS is higher in morcellation group than open surgery group (72.2\% vs $41.2 \%)$ [3], and 3-year overall survival rate is lower (64\% vs. 73\%) [4]. Therefore, preoperative diagnosis of ULMS, which can reduce the risk of tumor spread caused by morcellation, is of great significance.

However, it remains challenging in differentiating ULMS from uterine fibroid preoperatively. Ultrasonography is a preferred imaging modality, and ULMS characterizes unclear boundary, low resistance and high velocity blood flow. Unfortunately, ultrasonography has no definite diagnostic value due to great heterogeneity of ULMS. MRI performs slightly better, but does not facilitate a diagnosis with certainty. Furthermore, the great cost and limited access reduce its indications for uterine tumor.

Therefore, the objective of this study is to design a preoperative clinical characteristics scoring system for differentiating ULMS from uterine fibroid.

\section{Methods}

\section{Patients and study design}

This study retrospectively enrolled 45 ULMS patients receiving surgery in Peking Union Medical College Hospital, a complicated and severe cases referral center in China, from January 2013 to December 2018. The inclusion criteria were: a) postoperative pathological conformation of ULMS; b) initial treatment (no surgical intervention was performed previously); c) no evidence of tumor dissemination before surgery. 180 patients with uterine fibroid were enrolled in the control group, who were matched according to time of surgery ( \pm 1 year) and surgeons, at a ratio of 1:4. Patients with inflammatory disease or other malignant tumors were excluded.

\section{Data collection}

Patients' clinical data, ultrasonographic features, laboratory tests and surgical methods in ULMS group and control group were retrospectively retrieved. Ultrasonographic features included the largest diameter of uterus, number of fibroid and the largest diameter of fibroid. Laboratory tests included the number of neutrophil, lymphocyte, platelet, neutrophil to lymphocyte ratio (NLR), and serum lactate dehydrogenase (LDH) level. Ultrasonography and laboratory tests were performed within one month prior to surgery. This study was approved by the Institutional Review Board of Peking Union Medical College Hospital and written informed consents were obtained.

\section{Statistical analysis}

SPSS20.0 was used for data analysis. Continuous variables were compared by independent sample student $\mathrm{T}$ test, and categorized variables by Chi-square test or Fisher exact test. Multiple logistic regression analysis was performed to define risk factors of ULMS, which formed the scoring system. The scoring system was evaluated by ROC curve with sensitivity and specificity. A $p$ value of $<0.05$ was considered as statistical significance.

\section{Results}

\section{Incidence of occult ULMS}

From January 2013 to December 2018, 50 pathologically diagnosed ULMS patients received initial treatments in Peking Union Medical College Hospital. Of all, 5 cases were suspected as malignancy prior to operation due to evidence of tumor dissemination, and 45 cases were defined as occult ULMS. During the same period, 8169 uterine fibroid patients underwent myomectomies or hysterectomies (transabdominal, laparoscopic, transvaginal or hysteroscopic). The incidence of occult ULMS was $0.59 \%$ (95\% CI, 0.39-0.71\%).

\section{Patients characteristics}

45 patients in ULMS group and 180 patients in control group were enrolled in this study. The preoperative clinical characteristics of the two groups were shown in Table 1. Patients in ULMS group were older (47.0 vs 41.2 years old, $P<0.001$ ), with a higher postmenopausal rate $(20.0$ vs $5.0 \%, P=0.003)$. There were no significant differences in BMI, clinical manifestations (including abnormal vaginal bleeding and compression symptoms) between the two groups. Ultrasonographic manifestations showed that the maximum diameter of uterus $(9.6$ vs $8.0 \mathrm{~cm}, P=0.009)$ and the largest diameter of fibroids ( 7.8 vs $6.2 \mathrm{~cm}, P<0.001$ ) in ULMS group were significantly larger than control group. As to laboratory tests, LDH level (212.3 vs $169.1 \mathrm{U} / \mathrm{L}, P<0.001)$, number of neutrophil $\left(5.4\right.$ vs $\left.3.5 \times 10^{9} / \mathrm{L}, P<0.001\right)$ and platelet (323.4 vs $272.1 \times 10^{9} / \mathrm{L}, P=0.005$ ), NLR (3.3 vs $2.3, P=$ 0.007 ) in ULMS group were significantly higher than control group,. There were no significant differences in number of lymphocyte, hematocrit, CA125 and CA199 level.

\section{Risk factors of ULMS}

To differentiate ULMS from uterine fibroid, ROC curves of single factor, including age, the largest diameter of fibroid, LDH level, NLR and number of platelet were drawn respectively. The cutoff value of each factor was determined according to ROC curves, with age $\geq 40$ years old, tumor size $\geq 7 \mathrm{~cm}, \mathrm{LDH} \geq 193 \mathrm{U} / \mathrm{L}, \mathrm{NLR} \geq 2.8$ and number of platelet $\geq 298 \times 10^{9} / \mathrm{L}$.

Multivariate logistic regression analysis was performed to evaluate risk factors of ULMS. And Table 2 showed that age $\geq 40$ years old (OR 2.826, 95\%CI 1.326-5.461), tumor size $\geq 7 \mathrm{~cm}$ (OR 6.930, 95\% CI 2.872-16.724), 
Table 1 The preoperative clinical characteristics of ULSM group and control group

\begin{tabular}{|c|c|c|c|}
\hline & ULMS group $(N=45)$ & Control group $(N=180)$ & $P$ \\
\hline Age, yrs & $47.0 \pm 9.7$ & $41.2 \pm 7.7$ & $<0.001$ \\
\hline Postmenopausal & $9(20.0)$ & $9(5.0)$ & 0.003 \\
\hline Gravidity & $2.2 \pm 1.9$ & $1.9 \pm 1.4$ & 0.169 \\
\hline Parity & $1.1 \pm 0.6$ & $0.8 \pm 0.6$ & 0.008 \\
\hline $\mathrm{BMI}, \mathrm{kg} / \mathrm{m}^{2}$ & $23.0 \pm 2.9$ & $22.8 \pm 3.5$ & 0.801 \\
\hline Manifestations & $27(60.0)$ & $106(58.9)$ & 0.892 \\
\hline Abnormal uterine bleeding & $20(44.4)$ & $85(47.2)$ & 0.738 \\
\hline Compression symptoms & $4(8.9)$ & $23(12.8)$ & 0.612 \\
\hline Overall uterine size, cm & $9.6 \pm 2.6$ & $8.0 \pm 2.4$ & 0.009 \\
\hline Myoma size, cm & $7.8 \pm 2.3$ & $6.2 \pm 2.5$ & $<0.001$ \\
\hline$\geq 8 \mathrm{~cm}$ & 24 & 32 & $<0.001$ \\
\hline Solitary presumed myoma & 19 & 72 & 0.786 \\
\hline CA125, IU/L, in 140 cases & $30.0 \pm 39.6$ & $23.9 \pm 17.6$ & 0.218 \\
\hline CA199, IU/L, in 72 cases & $9.8 \pm 8.9$ & $20.2 \pm 31.0$ & 0.136 \\
\hline Neutrophil, $\times 10^{9} / \mathrm{L}$ & $5.4 \pm 3.2$ & $3.5 \pm 1.2$ & $<0.001$ \\
\hline Lymphocyte, $\times 10^{9} / \mathrm{L}$ & $1.8 \pm 0.6$ & $2.5 \pm 1.2$ & 0.669 \\
\hline NLR & $3.3 \pm 2.5$ & $2.3 \pm 1.1$ & 0.007 \\
\hline $\mathrm{HCT}, \%$ & $37.0 \pm 5.3$ & $37.6 \pm 3.8$ & 0.446 \\
\hline Platelet, $\times 10^{9} / \mathrm{L}$ & $323.4 \pm 112.2$ & $272.1 \pm 71.4$ & 0.005 \\
\hline $\mathrm{LDH}, \mathrm{U} / \mathrm{L}$ & $212.3 \pm 57.2$ & $169.1 \pm 27.0$ & $<0.001$ \\
\hline Initial surgery & & & $<0.001$ \\
\hline Total hysterectomy & 33 & 57 & \\
\hline Myomectomy & 12 & 123 & \\
\hline
\end{tabular}

Data was given as mean \pm SD or $\mathrm{n}(\%)$

ULSM uterine leiomyosarcoma, BMI body mass index, NLR neutrophil-to-lymphocyte ratio, $L D H$, lactate dehydrogenase, $H C T$ hematocrit

NLR $\geq 2.8$ (OR 3.032, 95\%CI 1.288-7.13), number of platelet $\geq 298 \times 10^{9} / \mathrm{L} \quad(\mathrm{OR} 3.688,95 \% \mathrm{CI} 1.452-9.266)$ and $\mathrm{LDH} \geq 193 \mathrm{U} / \mathrm{L}$ (OR 6.479, 95\%CI 2.658-15.792), were independent predictors of ULMS.

\section{A preoperative clinical characteristics scoring system} A preoperative clinical characteristics scoring system was designed based on OR values of the above factors. As shown in Table 3 and Fig. 1, tumor size $\geq 7 \mathrm{~cm}$,

Table 2 Risk factors of ULMS were evaluated by multivariate logistic regression analysis

\begin{tabular}{llll}
\hline Factor & $P$ value & OR & $95 \% \mathrm{Cl}$ \\
\hline Age $\geq 40 \mathrm{yrs}$ & 0.044 & 2.826 & $1.326-5.461$ \\
Postmenopausal & 0.122 & 2.642 & $0.770-9.068$ \\
Tumor size $\geq 7 \mathrm{~cm}$ & $<0.001$ & 6.930 & $2.872-16.724$ \\
$\mathrm{NLR} \geq 2.8$ & 0.011 & 3.032 & $1.288-7.139$ \\
Platelet $\geq 298 \times 109 / \mathrm{L}$ & 0.006 & 3.688 & $1.452-9.266$ \\
$\mathrm{LDH} \geq 193 \mathrm{U} / \mathrm{L}$ & $<0.001$ & 6.479 & $2.658-15.792$
\end{tabular}

ULSM uterine leiomyosarcoma, $N L R$ neutrophil-to-lymphocyte ratio, $L D H$ lactate dehydrogenase, $\mathrm{OR}$ odd ratio, $\mathrm{Cl}$ confidential interval
$\mathrm{LDH} \geq 193 \mathrm{U} / \mathrm{L}$ were assigned 2 points, while age $\geq 40$ years old, NLR $\geq 2.8$ and number of platelet $\geq 298 \times 10^{9} /$ $\mathrm{L}$ were assigned 1 point. The total score was 7 points, and each patient was scored according to the scoring system.

Scores of ULMS group and control group, together with sensitivities and specificities of different scores, were shown in Table 4. ROC curve of the scoring system was shown in Fig. 2. According to ROC curve, the

Table 3 A preoperative clinical characteristics scoring system for differentiating ULMS from fibroid

\begin{tabular}{llll}
\hline Score & 0 & 1 & 2 \\
\hline Age, yrs & $<40$ & $\geq 40$ & - \\
Myoma size, $\mathrm{cm}$ & $<7$ & - & $\geq 7$ \\
$\mathrm{NLR}$ & $<2.8$ & $\geq 2.8$ & - \\
$\mathrm{LDH}, \mathrm{U} / \mathrm{L}$ & $<193$ & - & $\geq 193$ \\
Platelet, $\times 109 / \mathrm{L}$ & $<298$ & $\geq 298$ & - \\
\hline
\end{tabular}

ULSM uterine leiomyosarcoma, $N L R$ neutrophil-to-lymphocyte ratio, $L D H$ lactate dehydrogenase 


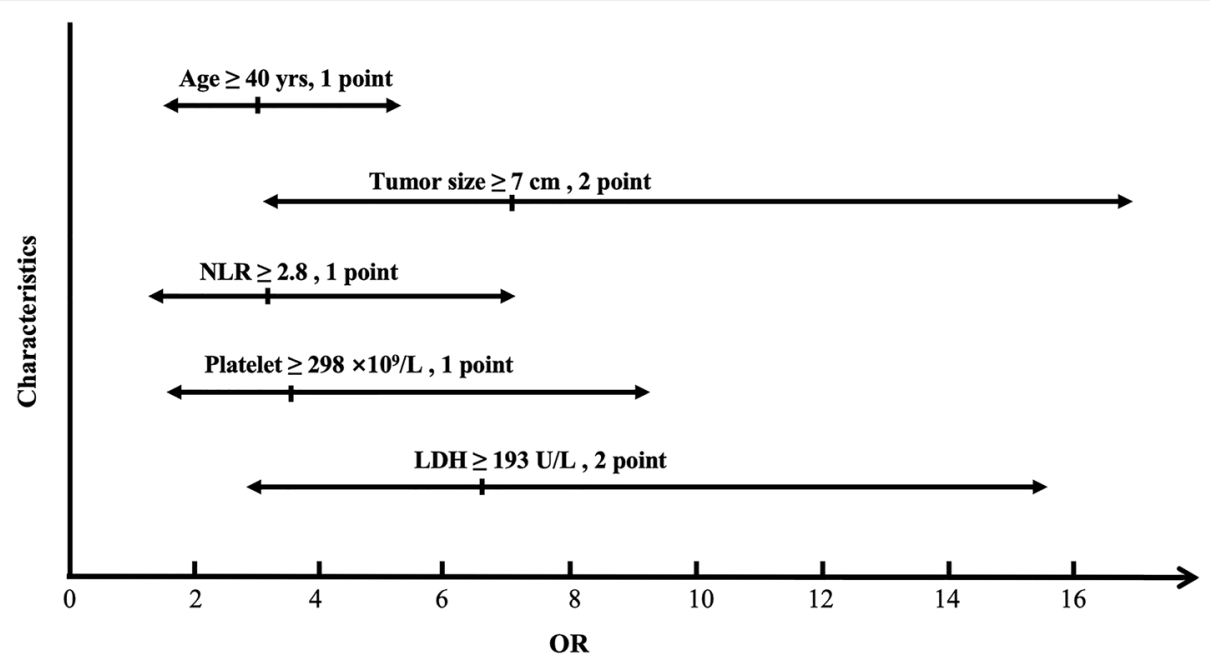

Fig. 1 The preoperative clinical characteristics scoring system was designed based on OR values of risk factors. Factors including tumor size $\geq 7$ $\mathrm{cm}, \mathrm{LDH} \geq 193 \mathrm{U} / \mathrm{L}$ were assigned 2 points, while factors including age $\geq 40$ years old, NLR $\geq 2.8$ and number of platelet $\geq 298 \times 10^{9} / \mathrm{L}$ were assigned 1 point. OR, odd ratio; LDH, actate dehydrogenase; NLR, neutrophil-to-lymphocyte ratio

optimal cutoff value was established at 4 points, with sensitivity of 0.800 and specificity of 0.778 in diagnosing ULMS from fibroid.

\section{Exploring efficacy of the scoring system among patients under $\mathbf{4 0}$ years old}

Patients under 40 years old with suspected uterine fibroid were more likely to retain uterus and received myomectomy due to fertility demands. Therefore, it was of greater clinical significance to distinguish ULMS from fibroid among patients under 40. In this study, there were 85 patients under 40, including 7 ULMS patients and 78 fibroid patients. The cutoff value of each risk factor was set according to ROC curves, with tumor size $\geq 7 \mathrm{~cm}, \quad \mathrm{LDH} \geq 189 \mathrm{U} / \mathrm{L}, \quad \mathrm{NLR} \geq 1.69$ and number of platelet $\geq 251 \times 10^{9} / \mathrm{L}$. Accordingly the scoring system was revised as: tumor size $\geq 7 \mathrm{~cm}$ and $\mathrm{LDH} \geq 189 \mathrm{U} / \mathrm{L}$ assigned 2 points, while NLR $\geq 1.69$ and number of platelet $\geq 251 \times 10^{9} / \mathrm{L}$ assigned 1 point. The total score was 6 points. 5 out of 7 ULMS patients and 9 out of 78 patients were scored $\geq 3$, with sensitivity of 0.714 and

Table 4 Scores of ULMS group and control group, and sensitivity, specificity and accuracy of different scores

\begin{tabular}{lllllllll}
\hline Scoring & $\geq 0$ & $\geq 1$ & $\geq 2$ & $\geq 3$ & $\geq 4$ & $\geq 5$ & $\geq 6$ & $\geq 7$ \\
\hline ULMS group & 45 & 45 & 45 & 39 & 36 & 25 & 16 & 6 \\
Control group & 180 & 156 & 118 & 66 & 40 & 11 & 2 & 0 \\
Total & 225 & 201 & 163 & 105 & 76 & 36 & 18 & 6 \\
Sensitivity & 1 & 1 & 1 & 0.867 & 0.800 & 0.556 & 0.356 & 0.133 \\
Specificity & 0 & 0.133 & 0.344 & 0.633 & 0.778 & 0.939 & 0.989 & 1 \\
Accuracy(\%) & 20.0 & 30.7 & 47.6 & 68.0 & 78.2 & 86.2 & 86.2 & 82.6 \\
\hline
\end{tabular}

ULSM uterine leiomyosarcoma specificity of 0.885 . Since the number of ULMS patients under 40 was limited in this study, larger sample researches were needed to validate the finding in future.

\section{Discussion}

In this study, the incidence of occult ULMS was $0.59 \%$. Multivariate logistic regression analysis showed that age $\geq 40$ years old, tumor size $\geq 7 \mathrm{~cm}, \mathrm{LDH} \geq 193 \mathrm{U} / \mathrm{L}$, NLR $\geq 2.8$ and number of platelet $\geq 298 \times 10^{9} / \mathrm{L}$ were independent predictors of ULMS. The preoperative clinical characteristic scoring system was designed according to the OR values of different risk factors, and scores $\geq 4$ points was an useful predictor in diagnosing ULMS from fibroid, with sensitivity of 0.800 and specificity of 0.778 .

Uterine sarcoma was a rare interstitial tissue derived malignant tumor, accounting for $3-7 \%$ of uterine malignant tumors. ULMS was the most common type, accounting for $63 \%$ of uterine sarcoma [5]. Clinical manifestations of ULMS were nonspecific, and most were found accidentally after hysterectomy or myomectomy. The incidence of occult ULMS was $0.0-0.64 \%$ (median 0.22\%) among presumed leiomyoma [6]. The study with the largest sample size till now reported that, rate of occult sarcoma was $0.36 \%$ (1/278) among 34,728 hysterectomies performed for presumed fibroids, and the rate of occult ULMS was $0.23 \%(1 / 429)$ [3]. Incidence of ULMS in this study was $0.59 \%$, consistent with previous reports.

Age was a predictor of ULMS. A multicenter retrospective study reported 221 patients of ULMS and 81 patients of endometrial stromal sarcoma were diagnosed at an average age of 55 years old, and 59\% were postmenopausal [7]. Hosh et al. found that the age-adjusted incidence rate for patients aged 50 years or older was 


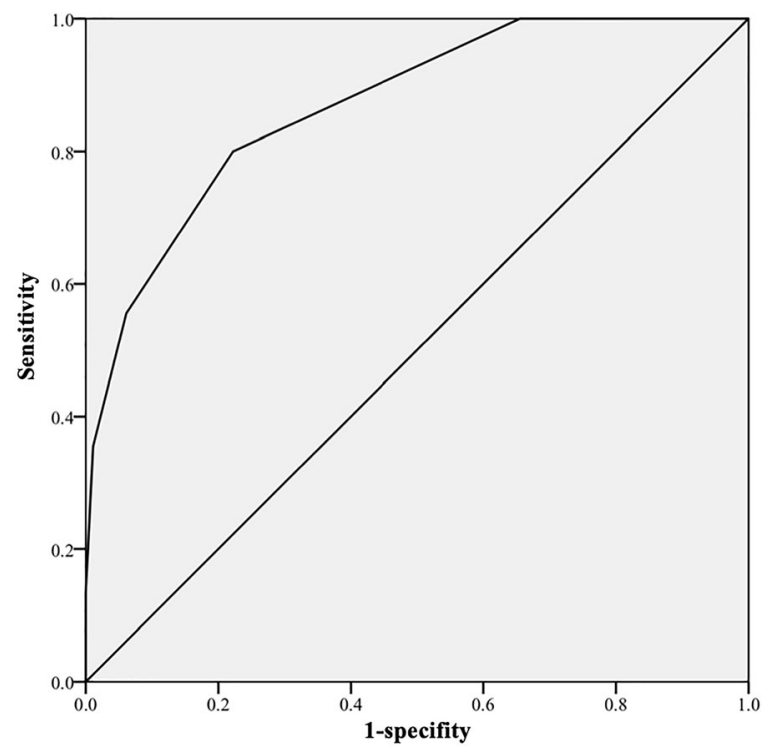

Fig. 2 ROC curve of the scoring system was shown. The optimal cutoff value was established at 4 points, with sensitivity of 0.800 and specificity of 0.778

higher than younger patients $(6.4 / 10$ vs $1.5 / 10, P<$ 0.0001), based on Surveillance, Epidemiology, and End Results (SEER) database [8]. In this study, patients in ULMS group were older ( 47.0 vs 41.2 years old, $P<$ 0.001 ) than control group, and age $\geq 40$ years old (OR 2.826, 95\% CI 1.326-5.461) was an independent predictor of ULMS.

Tumor size might be a predictor of ULMS. In 2017, the European Society of Gastrointestinal Endoscopy (ESGE) confirmed that certain signs could cause suspicion of sarcoma, including a lesion exceeding $8 \mathrm{~cm}$ [9]. In a study of 15 ULMS patients, tumor size $>7 \mathrm{~cm}$ (adjusted-OR 0.973; 95\% CI, 0.75-1.26; $P=0.08$ ) might be a predictor of ULMS [10]. Another study involving 31 uterine sarcoma patients showed that large tumor size $(>8.0 \mathrm{~cm})$ was an independent risk factor $(P=0.048)$ [11]. Results in this study also indicated that tumor size of ULMS was significantly larger than fibroid (7.8 vs 6.2 $\mathrm{cm}, P<0.001$ ), and tumor size $\geq 7 \mathrm{~cm}$ was an independent predictor of ULMS (OR 6.930, 95\% CI 2.87216.724). However, some studies had opposite results. Chen's study with 66 cases of uterine sarcoma showed no difference in size between uterine sarcoma and fibroid ( 9.6 vs $8.5 \mathrm{~cm}, P=0.40$ ) [12].

Recent studies had confirmed that NLR might a markers for diagnosis and prognosis of malignant tumors. It was generally believed that inflammation was involved in the process of tumor proliferation, angiogenesis, metastasis and therapeutic response. The concentration of inflammatory cells in tumor microenvironment was high. Some secondary hematological changes might occur in tumor patients, including lymphocyte decrease, neutrophil and platelet elevation. In recent years, studies had found that NLR and platelet to lymphocyte ratio (PLR) might be markers for diagnosis and prognosis of colorectal cancer, ovarian epithelial cancer, liver cancer, pleural malignant mesothelioma, non-small cell lung cancer and breast cancer [13, 14]. Moreover, a meta-analysis enrolling 2820 patients in 14 studies showed that NLR before treatment was associated with the prognosis of soft tissue sarcoma [15]. A few studies focused on the diagnostic value of NLR in uterine sarcoma. Kim compared NLR with serum CA125 as preoperative diagnostic markers for uterine sarcoma, and NLR $(>2.12$ ) was found to be more accurate (sensitivity, 74.5\%; specificity, 70.3\%) [16]. A study of 31 cases of ULMS, endometrial stromal sarcoma and undifferentiated sarcoma showed that NLR $>2.1(P=0.041)$ was an independent predictor of uterine sarcoma [11]. In this study enrolling 45 ULMS patients, number of neutrophil (5.4 vs $\left.3.5 \times 10^{9} / \mathrm{L}, P<0.001\right)$, NLR (3.3 vs $\left.2.3, P=0.007\right)$ and number of platelet (323.4 vs $\left.272.1 \times 10^{9} / \mathrm{L}, P=0.005\right)$ increased significantly; and NLR $\geq 2.8$ (OR 3.032, 95\%CI 1.288-7.139) and number of platelet $\geq 298 \times 10^{9} / \mathrm{L}$ (OR $3.688,95 \% \mathrm{CI}$ 1.452-9.266) were independent predictors of ULMS.

LDH level was one of the markers in cancer diagnosis and prognosis. Metabolism of cancer cells was characterized by increased glucose uptake and lactic acid production. LDH reversibly catalyzed the conversion of pyruvate to lactate or lactate to pyruvate. Elevated LDH level was a negative prognostic biomarker not only because it was a key enzyme involved in cancer metabolism, but also it allowed neoplastic cells to suppress and evade the immune system by altering tumor microenvironment [17]. An immunohistochemical stain study of 50 uterine sarcoma and 26 fibroids revealed that the positivity rates for LDH-A and LDH-D were significantly higher in patients with uterine sarcoma $(P<0.05)$ [18]. Nagai summarized 15 cases of uterine sarcoma and showed that $\mathrm{LDH}>279 \mathrm{U} / \mathrm{L}$ was an independent predictor in the diagnosis of uterine sarcoma [19]. LDH was also used in the diagnosis of other gynecological tumors. A study involving 20 cases found that LDH might be one of the diagnostic markers for ovarian epithelial cancer $(\mathrm{AUC}=0.77)$ [20]. In addition, LDH was considered as a prognostic factor for sarcoma. LDH level before treatment was an effective marker of the prognosis of Ewing's sarcoma [21]. In this study, LDH level in ULMS group was higher $(212.3$ vs $169.1 \mathrm{U} / \mathrm{L}, P<0.001)$ than control, and LDH $\geq 193 \mathrm{U} / \mathrm{L}$ (OR 6.479, 95\%CI 2.65815.792) was an independent predictor of ULMS.

Due to the limited diagnostic value of single factor in ULMS, a few researchers had explored scoring systems incorporating multiple factors to increase diagnostic value. Nagai enrolled 9 patients with ULMS, 3 patients with adenosarcoma and 3 patients with endometrial 
stromal sarcoma [22]. A revised pre-operative sarcoma scoring system was designed, including age, serum LDH level and cytological finding, with a maximum score of 10 points and an optimal cut-off value of 4 points. The accuracy, positive predictive value, and negative predictive value were 93.7, 92.3 and $94.0 \%$, respectively. Since adenosarcoma and endometrial stromal sarcoma were more easily detected by cytological examinations than ULMS, the results of this study were perhaps not suitable for ULMS. In Cho's study, sarcoma index was calculated by summing the number of risk factors. Higher sarcoma index was associated with increased risk of uterine sarcoma $(0,13.6 \% ; 1,21.7 \% ; 2,62.5 \% ; 3,100 \%)$ [11]. In this study, according to the OR values of different risk factors, a preoperative clinical characteristics scoring system was designed, including factors of age $\geq 40$ years old, tumor size $\geq 7 \mathrm{~cm}, \mathrm{LDH} \geq 193 \mathrm{U} / \mathrm{L}, \mathrm{NLR} \geq 2.8$ and number of platelet $\geq 298 \times 10^{9} / \mathrm{L}$, with optimal cutoff value 4 points, sensitivity 0.800 and specificity 0.778 .

As far as we knew, this was the largest sample study focusing on preoperative diagnosis of ULMS. The scoring system promoted to preoperative diagnosis of ULMS and reduced risk of dissemination of ULMS caused by morcellation. The main limitation of this study was it was a single center based retrospective study. And multicenter studies with larger sample size were needed to confirm the relevant conclusions.

\section{Conclusion}

The incidence of occult ULMS was low and preoperative diagnosis was difficult. Age $\geq 40$ years old, tumor size $\geq 7$ $\mathrm{cm}, \mathrm{LDH} \geq 193 \mathrm{U} / \mathrm{L}, \mathrm{NLR} \geq 2.8$ and number of platelet $\geq 298 \times 10^{9} / \mathrm{L}$ were independent predictors of ULMS. The ULMS preoperative clinical characteristics scoring system could be helpful in preoperative diagnosis of occult ULMS.

\section{Abbreviations \\ ULMS: Uterine leiomyosarcoma; NLR: Neutrophil to lymphocyte ratio; LDH: Lactate dehydrogenase}

\section{Acknowledgements}

None.

\section{Authors' contributions}

$\mathrm{GZ}, \mathrm{XY}$, and $\mathrm{J}$ conceived and designed the research; GZ, QF and HS collected and analyzed the data; GZ and LZ conducted the statistical analysis; GZ and XY prepared the manuscript. All authors have read and approved the final manuscript.

\section{Funding}

The study was supported by the National Natural Science Foundation of China (81830043). The fund had no role in study design, subject enrollment, or data analysis.

\section{Availability of data and materials}

The datasets used in the current study available from the corresponding author on reasonable request.

\section{Ethics approval and consent to participate}

The study was approved by the Institutional Review Board of Peking Union Medical College Hospital. And written informed consents were obtained.

\section{Consent for publication}

Not applicable.

\section{Competing interests}

The authors declare that they have no conflicts of interest.

Received: 8 October 2019 Accepted: 25 May 2020

Published online: 03 June 2020

\section{References}

1. D'Angelo E, Prat J. Uterine sarcomas: a review. Gynecol Oncol. 2010;116(1): 131-9.

2. Park JY, Park SK, Kim DY, Kim JH, Kim YM, Kim YT, et al. The impact of tumor morcellation during surgery on the prognosis of patients with apparently early uterine leiomyosarcoma. Gynecol Oncol. 2011;122(2):255-9.

3. Raine-Bennett T, Tucker LY, Zaritsky E, Littell RD, Palen T, Neugebauer R, et al. Occult uterine sarcoma and Leiomyosarcoma: incidence of and survival associated with Morcellation. Obstet Gynecol. 2016;127(1):29-39.

4. George S, Barysauskas C, Serrano C, Oduyebo T, Rauh-Hain JA, Del Carmen $M G$, et al. Retrospective cohort study evaluating the impact of intraperitoneal morcellation on outcomes of localized uterine leiomyosarcoma. Cancer. 2014;120(20):3154-8.

5. Trope CG, Abeler VM, Kristensen GB. Diagnosis and treatment of sarcoma of the uterus. Rev Acta Oncol. 2012;51(6):694-705.

6. Wong M, De Wilde RL, Isaacson K. Reducing the spread of occult uterine sarcoma at the time of minimally invasive gynecologic surgery. Arch Gynecol Obstet. 2018;297(2):285-93.

7. Wais M, Tepperman E, Bernardini MQ, Gien LT, Jimenez W, Murji A. A multicentre retrospective review of clinical characteristics of uterine sarcoma. J Obstet Gynaecol Can. 2017;39(8):652-8.

8. Zhao WC, Bi FF, Li D, Yang Q. Incidence and clinical characteristics of unexpected uterine sarcoma after hysterectomy and myomectomy for uterine fibroids: a retrospective study of 10,248 cases. Onco Targets Ther. 2015:8:2943-8.

9. Halaska MJ, Haidopoulos D, Guyon F, Morice P, Zapardiel I, Kesic V, et al. European Society of Gynecological Oncology Statement on fibroid and uterine Morcellation. Int J Gynecol Cancer. 2017;27(1):189-92.

10. Oduyebo T, Hinchcliff E, Meserve EE, Seidman MA, Quade BJ, Rauh-Hain JA, et al. Risk factors for occult uterine sarcoma among women undergoing minimally invasive gynecologic surgery. J Minim Invasive Gynecol. 2016; 23(1):34-9.

11. Cho HY, Kim K, Kim YB, No JH. Differential diagnosis between uterine sarcoma and leiomyoma using preoperative clinical characteristics. J Obstet Gynaecol Res. 2016:42(3):313-8.

12. Chen I, Firth B, Hopkins L, Bougie O, Xie RH, Singh S. Clinical Characteristics Differentiating Uterine Sarcoma and Fibroids. JSLS. 2018;22(1):e2007.00066.

13. Fest J, Ruiter R, Mulder M, Groot Koerkamp B, Ikram MA, Stricker BH, et al. The systemic immune-inflammation index is associated with an increased risk of incident cancer-A population-based cohort study. Int J Cancer. 2020; 146(3):692-8.

14. Huang H, Liu Q, Zhu L, Zhang Y, Lu X, Wu Y, et al. Prognostic value of preoperative systemic immune-inflammation index in patients with cervical Cancer. Sci Rep. 2019;9(1):3284

15. Liu G, Ke LC, Sun SR. Prognostic value of pretreatment neutrophil-tolymphocyte ratio in patients with soft tissue sarcoma: a meta-analysis. Medicine (Baltimore). 2018;97(36):e12176.

16. Kim HS, Han KH, Chung HH, Kim JW, Park NH, Song YS, et al. Neutrophil to lymphocyte ratio for preoperative diagnosis of uterine sarcomas: a casematched comparison. Eur J Surg Oncol. 2010;36(7):691-8.

17. Ding J, Karp JE, Emadi A. Elevated lactate dehydrogenase (LDH) can be a marker of immune suppression in cancer: interplay between hematologic and solid neoplastic clones and their microenvironments. Cancer Biomark. 2017;19(4):353-63.

18. Song KJ, Yu XN, Lv T, Chen YL, Diao YC, Liu SL, et al. Expression and prognostic value of lactate dehydrogenase-a and -D subunits in human uterine myoma and uterine sarcoma. Medicine (Baltimore). 2018;97(14):e0268. 
19. Nagai T, Takai Y, Akahori T, Ishida H, Hanaoka T, Uotani T, et al. Novel uterine sarcoma preoperative diagnosis score predicts the need for surgery in patients presenting with a uterine mass. Springerplus. 2014;3:678.

20. Bastani A, Asghary A, Heidari MH, Karimi-Busheri F. Evaluation of the sensitivity and specificity of serum level of prostasin, CA125, LDH, AFP, and hCG+beta in epithelial ovarian cancer patients. Eur J Gynaecol Oncol. 2017; 38(3):418-24.

21. Li S, Yang Q, Wang H, Wang Z, Zuo D, Cai Z, et al. Prognostic significance of serum lactate dehydrogenase levels in Ewing's sarcoma: a meta-analysis. Mol Clin Oncol. 2016;5(6):832-8.

22. Nagai T, Takai Y, Akahori T, Ishida H, Hanaoka T, Uotani T, et al. Highly improved accuracy of the revised PREoperative sarcoma score (rPRESS) in the decision of performing surgery for patients presenting with a uterine mass. Springerplus. 2015;4:520.

\section{Publisher's Note}

Springer Nature remains neutral with regard to jurisdictional claims in published maps and institutional affiliations.

Ready to submit your research? Choose BMC and benefit from:

- fast, convenient online submission

- thorough peer review by experienced researchers in your field

- rapid publication on acceptance

- support for research data, including large and complex data types

- gold Open Access which fosters wider collaboration and increased citations

- maximum visibility for your research: over $100 \mathrm{M}$ website views per year

At BMC, research is always in progress.

Learn more biomedcentral.com/submissions 